\title{
Determine the Gamma Radiation Technique to Sterilization of Insects from Risk Factor
}

\author{
Baye Zinabe Kebede \\ Department of Physics, College of Natural and Computational Science, Mekdela Amba University, Mekdela Amba, Ethiopia \\ Email address: \\ bayezinabe82@gmail.com \\ To cite this article: \\ Baye Zinabe Kebede. Determine the Gamma Radiation Technique to Sterilization of Insects from Risk Factor. World Journal of Applied \\ Physics. Vol. 6, No. 2, 2021, pp. 35-40. doi: 10.11648/j.wjap.20210602.12
}

Received: June 3, 2021; Accepted: June 29, 2021; Published: July 6, 2021

\begin{abstract}
The sterile insect technique (SIT) is an environment-friendly blighter management technique with application within the area-wide integrated management of key pests, together with the suppression or elimination of introduced populations and therefore the exclusion of recent introductions. Procreative sterility is often evoked by radiation, a convenient and consistent technique that maintains an inexpensive degree of aggressiveness within the discharged insects. The price and effectiveness of an effective program integration of the SIT rely on the balance between sterility and aggressiveness, but it seems that current operational programs with an associate SIT element aren't achieving an associated, applicable balance. In this paper, we will discuss how to improve the sterilization method as well as a simple model and procedure for determining the optimum dose. The work in the gift was focused on an association analysis of the electromagnetic wave technique for insect sterilization as a risk issue. Discharge of enormous numbers of gamma-radiated male flies over the complete island caused concern regarding sterilization as an attainable technique for the dominant insects' public health. Several studies on the sterilization of varied insect vectors of sickness have been conducted since then. The study's goal was to check if electromagnetic waves were an efficient insect management mechanism. Insect sterilization is commonly used to create safe environments for fruit homes, manage blighters, and avoid livestock health issues. The sterilization insect technique entails mass-rearing of the target species, sterilization of the male, and unharnessing in sufficient numbers to equal wild male flies in the natural environment. It's species-specific, with no results for any of the 'non-target' species.
\end{abstract}

Keywords: Gamma Radiation, Sterilization, Insect, Irradiation and Effect

\section{Introduction}

Controlling methods available, depending on the goal of the sterilization and thus the material sterilized [1]. The sterilization technique chosen depends on the materials and devices used to ensure no harm [2]. Radiation delivers a precise dose that can take anywhere between minutes to hours, depending on the thickness and volume of the product [3]. Beam irradiation will provide an equivalent dose for a few seconds, but it will only be for small items [4]. Electromagnetic waves are the most basic method for sterilizing insects. a result, insects gain radiotolerance as they develop, and since several process stages of blighter may be found in recently shipped artifacts, it's important to know the radiation sensitivity of stages of the target insect before instituting radiation quarantine treatments [5]. The term radiation includes radio waves, radar, infrared, microwaves, ultraviolet, electrons, X-rays and gamma-rays. Radiation is also used as a direct treatment against stored-product pests as fumigants and alternative management ways or by genetic management involving the discharge of irradiated sterile males so most of the females can lay sterile eggs [6]. Irradiation of male insects might end in dominant deadly mutations within the spermatozoan, killing spermatogonia cells, inactivation of the spermatozoan, and weakening of the males. Reckoning on the dose and age or physiological state of the insect, irradiation of the actively growing stages of the Associate in Nursing insect will have many effects [7]. The majority of genetic sterility is caused by irradiation, which results in failure of spermatozoan bundles to separate, lack of motility within the spermatozoa, failure of the spermatozoan to maneuver through the spermatheca, or other malfunctions that may stop copying and can be induced in either sex by selecting the appropriate dose and biological process stage 
[8]. Control of insect species for crop and livestock protection, as well as the prevention of diseases such as infectious diseases. Pesticides, for example, have fallen out of favor due to their indiscriminate effects as well as negative and unpredictable effects on the environment [9].

\section{Literature Review}

\subsection{Property of Gamma Radiation}

Because of its deep penetration power and low rate, gamma irradiation is thought to be an extremely common application of nuclear technology for peaceful purposes, such as a physical agent of sterilization or removal, with high potency in killing microorganisms by breaking the valency bonds of microorganism deoxyribonucleic acid and viruses [10]. Electromagnetic radiation has been thought to be a secure, price-competitive methodology for the sterilization of aid merchandise, extension of shelf-life quality improvement, and reduction of bioburden in food merchandise. What is more, exposure to electromagnetic radiation will offer attainable solutions for the treatment of contaminated waters (municipal, industrial waste) aiming at the development of their chemical and biological quality [11]. Though there are numerous sterilization methods that correlate with the goal of sterilization and thus the material that can be sterilized, there are some common sensible options for exploitation of electromagnetic radiation that include: precise dosing, quick process, uniform dose distribution, system flexibility, measures unleashed, and the immediate availability of products on the market. When a biological material is irradiated, molecular bonds are broken, ions are created, and free radicals are fashioned. Free radicals attack additional molecular bonds, and once deoxyribonucleic acid is broken, it will cause the formation of dominant deadly mutations within the germ cells. Injury to corporeal cells additionally happens, particularly in cells undergoing cellular division [10]. In general, injury to the germ and corporeal cells will increase with dose and corporeal injury decreases once irradiated later in the development of the insect, because the range of cells undergoing division decreases. As field aggressiveness could be a crucial parameter, it's vital to attenuate the adverse effects of irradiation. Though it is commonly assumed that free males must be forced to be completely sterile, it has been suggested that a significant amount of sterility will be introduced into the human population through lower radiation doses with a large number of competitive insects. Moreover, reduced aggressiveness will be partially overcome by increasing the magnitude relationship of sterile-to-wild insects [12].

\subsection{Principal of Gamma Irradiation}

Radiation has an impact on all living things square measures are primarily related to chemical changes, but they are also linked to physical and physiological factors. The physical parameters are measured by dose rate, dose distribution, and radiation quality square. The most vital physiological and environmental parameters measure temperature, water content and concentration. The action of radiation on living organisms is often divided into direct and indirect effects [15]. Complete obliteration of those microorganisms (sterilization) is crucial to the security of medical devices and pharmaceutical products. The sterilization method must be valid in order to ensure that it effectively and consistently kills any microorganisms that may be present in the pre-sterilized product. Radiation sterilization, as a physical cooling method, has been widely employed in several developed and developing countries for the sterilization of health care products. Earlier, a minimum dose of twentyfive kGy was habitually applied to several medical devices, pharmaceutical products and biological tissues. Now, according to the International Organization for Standardization (ISO), the sterilization dose should be determined for each type of product based on its bioburden [8]. In general, the primary manufacturer of the medical product is responsible for determining the sterilization dose, and the UN agency should have access to a qualified biological science laboratory.

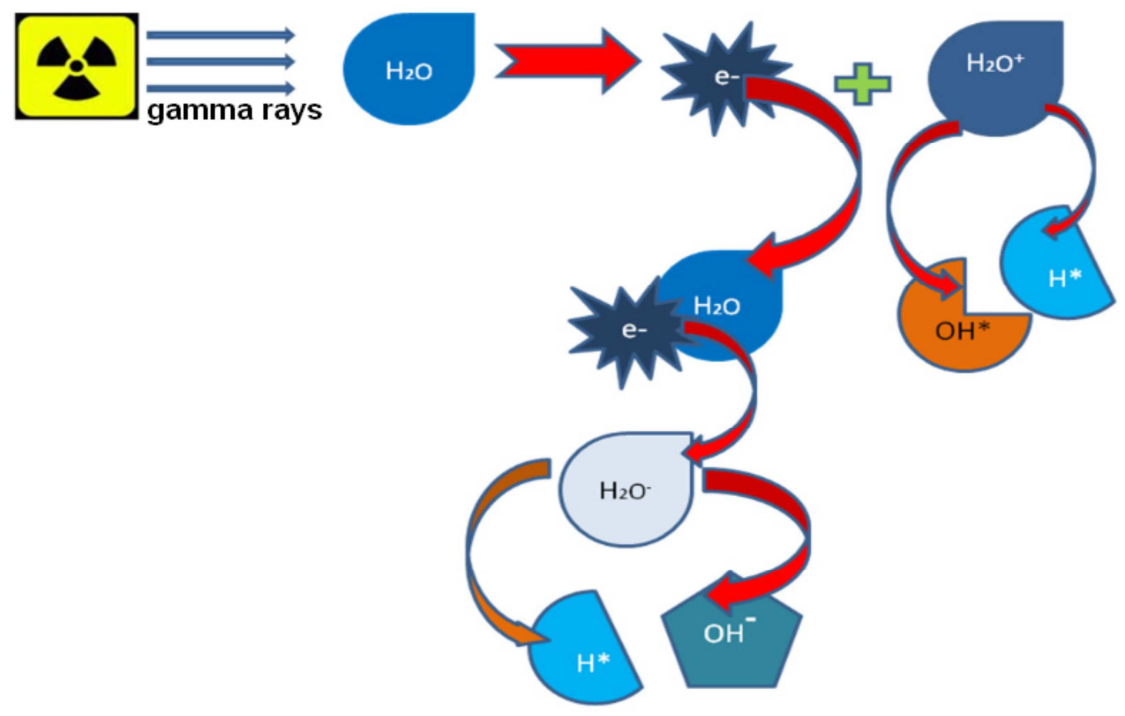

Figure 1. The possible effect of gamma radiation to ward water molecule [8]. 
A thorough investigation has been conducted into the lethal impact of radiation on microorganisms, as measured by the loss of cells' colony-forming ability in the nutrient medium. A lot of progress has been made in identifying the mechanism of inactivation. There still remains a lot of doubt about the character of the crucial lesions involved, although it seems that unwholesomeness is primarily the consequence of genetic injury [13]. Many hypotheses have been proposed and tested regarding the mechanism of radiation-induced cell injury. Some scientists have proposed a mechanism known as 'radio toxins,' which are harmful substances produced at regular intervals that are irradiated into cells and can cause death. Nuclear techniques might play a crucial role in enabling the economical production of helpful insects to be utilized in augmentative biological management. There are important opportunities for increasing the use of classical and augmentative biological management through nuclear techniques for the assembly and unleashing of biological management agents [14]. Once radiation passes through materials, it breaks chemical bonds. The radiation method has been used commercially for nearly forty years. Nonparticulate radiation from $60 \mathrm{Co}$, lepton beams and x-rays, as well as space units, are all capable of sterilizing medical devices used in operations and various aid treatments. Radiation is employed to sterilize implants, artificial joints, syringes, blood bags, gowns, bottle teats for newborn units, and dressings [15]. The Sterile Insect Technique, additionally referred to as the Sterile Male Technique, consists primarily of rearing and emotional male insects that are rendered sexually sterile by exposure to a radiation supply in massive numbers bigger than those found within the natural atmosphere [16]. The continuing unleashing of sterile males will increase the quantitative relationship of sterile to ancients, leading to species suppression or extinction [17]. The practice of sterile male insects is horrifying in terms of natural population management.

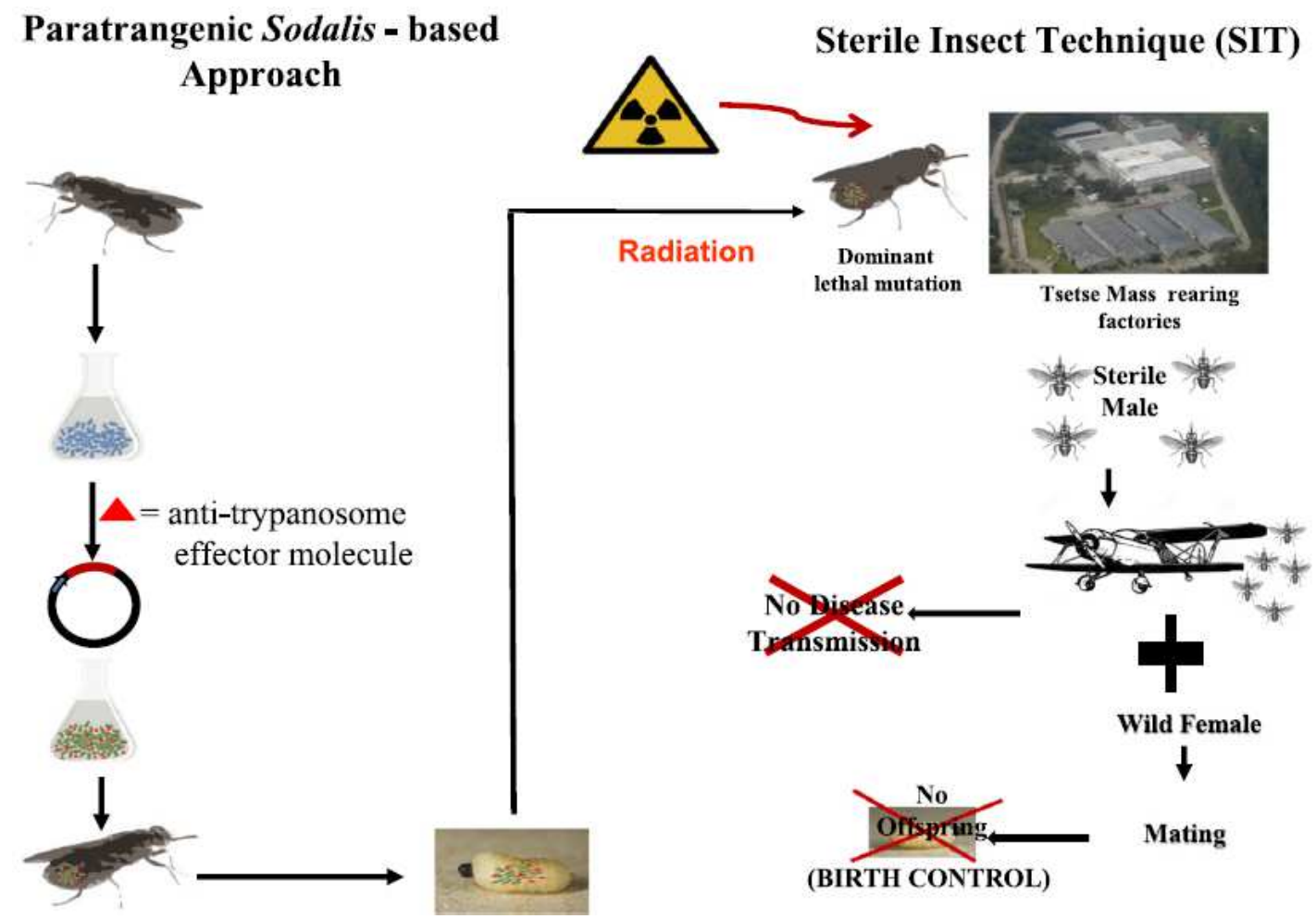

Figure 2. Sterilization of insect by irradiation [10].

\section{Method of Insect Sterilization}

In the current work, we've used logical and technical reviews taken from the previous 10 analysis articles from 2010-2020 G. C. We gathered the necessary information by comparing and distinguishing the insect stage once before employing the sterilization technique. Gamma ray techniques are used to control the mechanism of insects that break fruit. Gamma rays were radiation emitters with a high penetration power. In this case, we tend to evaluate previous work that resulted in sterilization by emitting radiation toward the insect's habitats. Significantly, we intend to eliminate the downside exposure risk to the health and safety of animals, fruit, and the soul. The most common sterilization technique is the use of radiation from $60 \mathrm{Co}$ or $137 \mathrm{Cs}$. The sterilizing dose is a critical component for the cost-effective application of SIT. This should be kept to a minimum in order to preserve the insect's behavior. Laboratory studies on three experimental groups of tsetse fly flies at completely different doses (60Gy, 80Gy, 100Gy, and 120Gy) on mated fertile females revealed that the number of aborted eggs/larvae was directly proportional to the dose $[3,11]$. 


\section{Result of Insect Sterilization}

As we've seen, the application of the Sterile Insect Technique entails the production, sterilization and ulterior unharness of sterile male insects into a target population in an integrated space-wide management strategy of mosquitoes. Free males inseminate wild females with sterile sperm cells. The females fail to provide viable offspring, resulting in an overall size reduction of the target population. Sterilization by irradiation has long been recognized as the most practical method of insect control. The optimum dose of radiation is needed to provide potent males that are compatible with a union with wild females. We tend to do so, testing numerous radiation doses at the immature stage. And determination was created as the result of radiation doses in adult emergence. In our results, the 60 Gy dose was found optimum for the specified variety of union, no or terribly low fecundity and hatching and so could also be thought-about within the Sterile Insect Techniques reported similar necessities for sterilization of insect techniques of mosquitoes. They emphasized the high level of stability between sterile males and wild females. Sterile males ought to be compatible with a union with wild women under field conditions. The utilization of SIT as an insect management strategy has been reported by some earlier researchers. The use of SIT as a safe technique in conjunction with accumulation production, release, and subsequent union aggressiveness with wild females indicates that SIT is an insect tormentor management methodology with excellent success against agricultural insect pests.

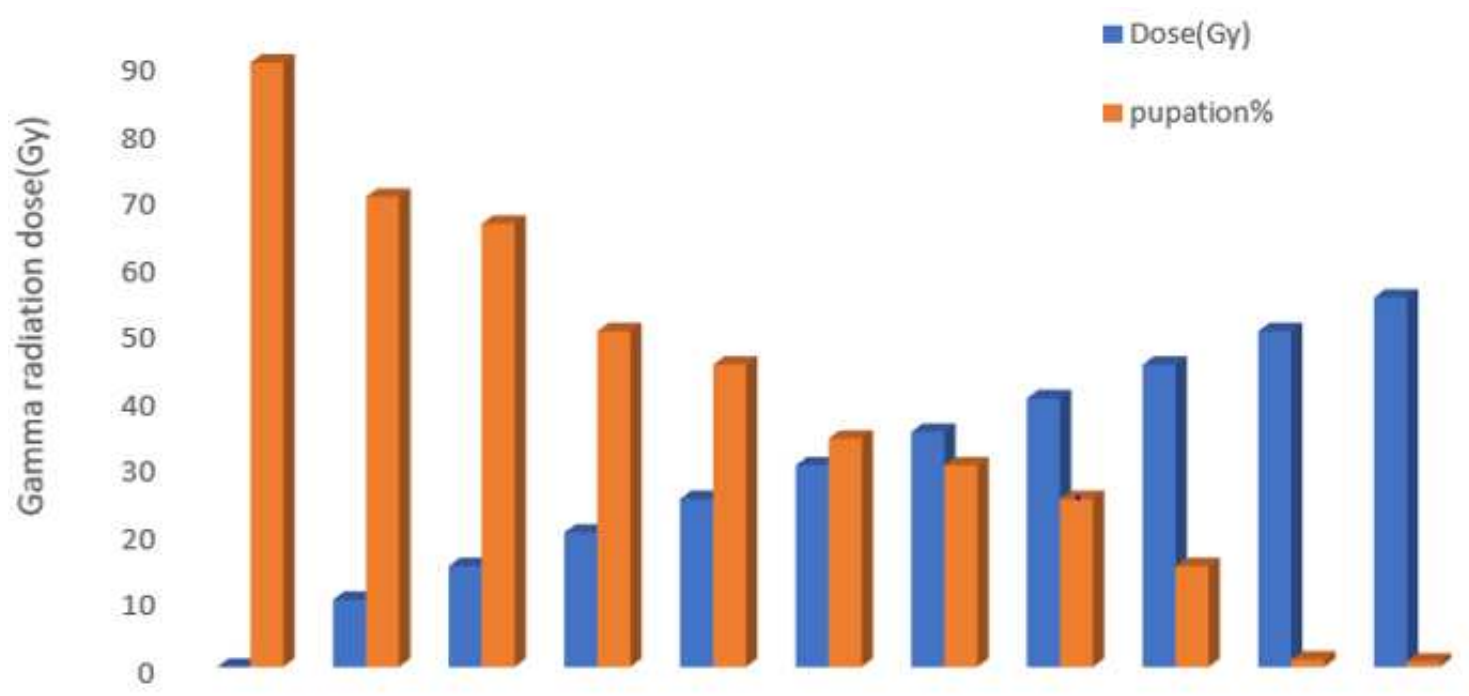

The distribution of insect pupa stage

Figure 3. Effect of gamma radiation to controlling insect from any risk to human being [3, 11$]$.

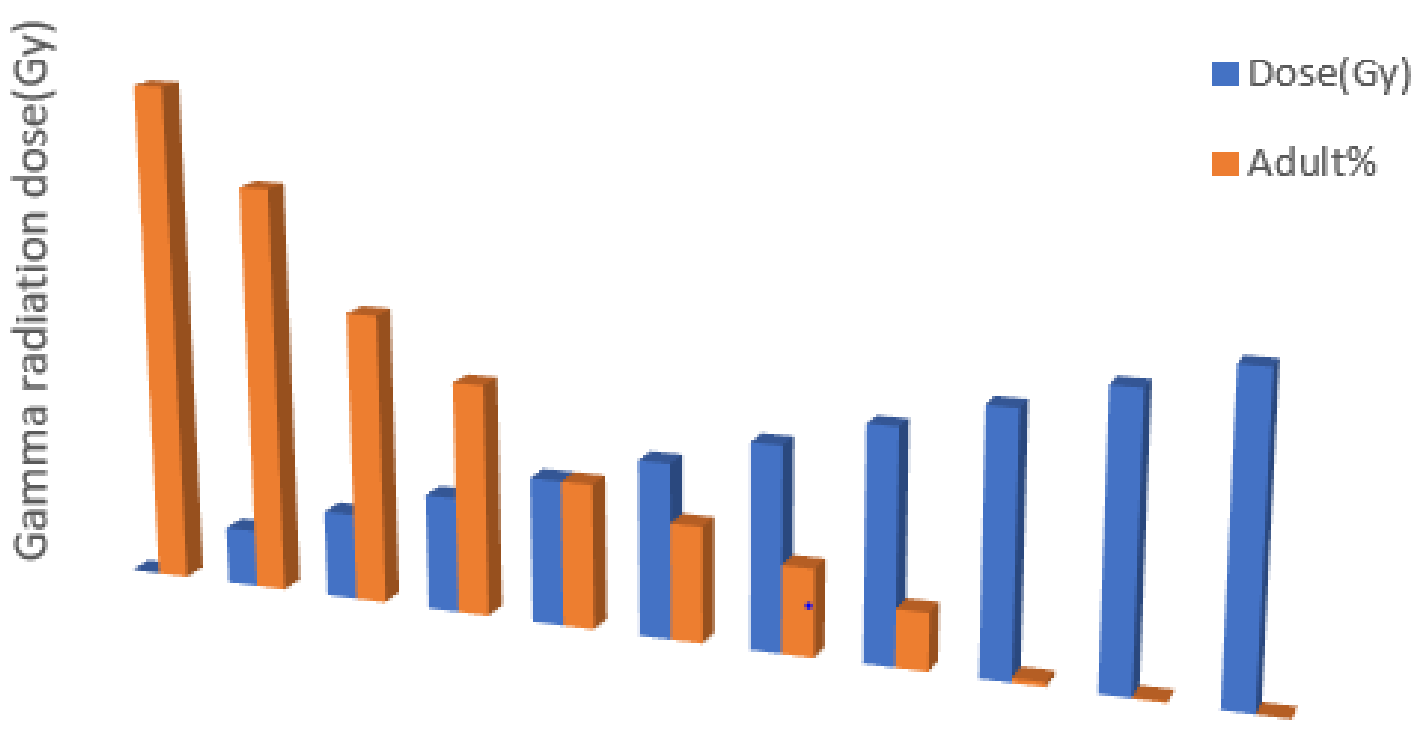

The distribution of insect adult stage

Figure 4. Checking the radiation effect control mechanism within adult stage [3, 11]. 


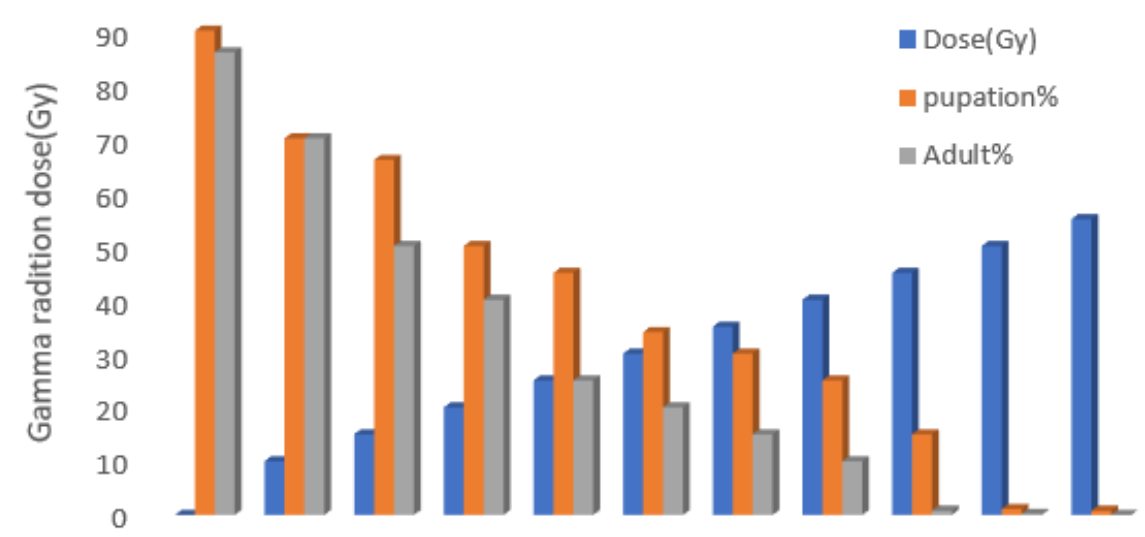

The distribution of both pupation with adult insect stage

Figure 5. Comparison the effect controlling mechanism of gamma radiation both at pupa and larva stage [3, 11].

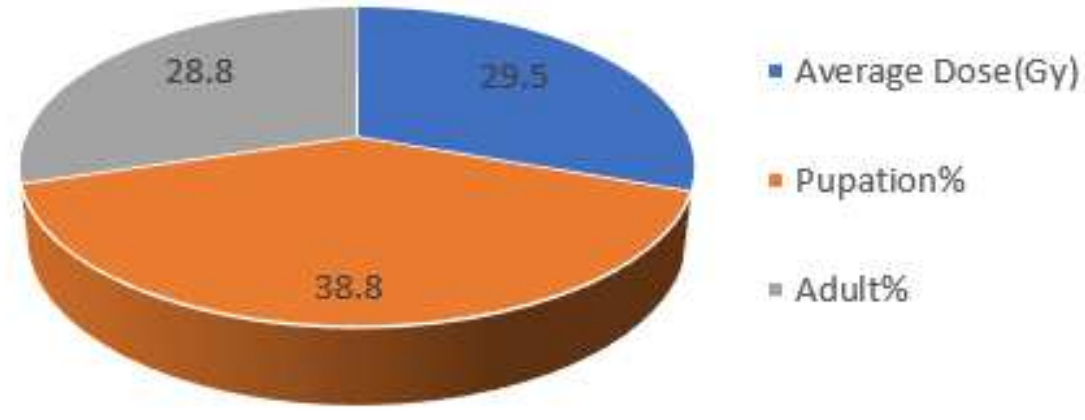

Figure 6. Pi-chart of comparison the effect average dose radiation on adult and pupa stage.

When we compared the average dose rate of pupa with adult insect which more easily controlled than that of pupa stage. This demonstrates that gamma radiation is an effective monitoring mechanism for sterilizing insects from fruit, crops, and livestock.

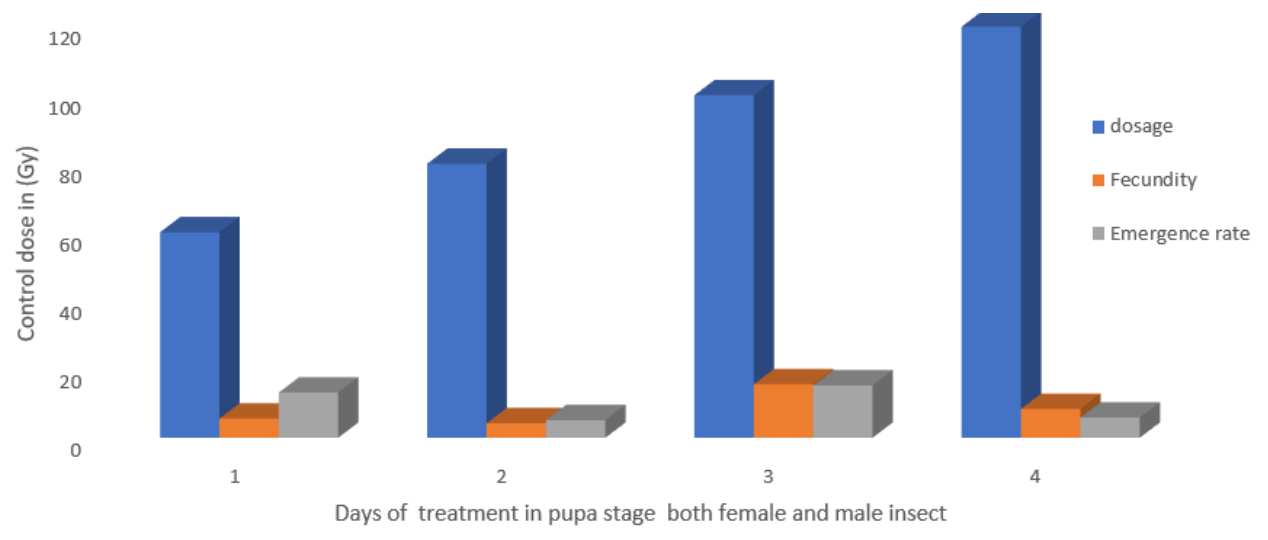

Figure 7. The mean number of pupas produced by female after mating with treated male against gamma dose of irradiation [3, 11].

As shown at the top of figure 5, studies on three experimental groups of tsetse fly flies at completely different doses (60Gy, 80Gy, 100Gy, and 120Gy mated fertile females) revealed that the number of aborted eggs/larvae was directly proportional to the dose. Females mated with males irradiated at higher doses were more economical and resulted in high abortion of eggs/larvae, reduction of pupae and reduced the quantity of emerged adults from pupae than the lower irradiation doses. Furthermore, the number of pupae produced and adults born from pupae was lower in groups where sterile males were stacked on top of fertile males, and the effect was reciprocally proportional. On the other hand, teams containing a bigger range of fertile male ratios than sterile males had a bigger range of blocked pupae.

\section{Conclusion}

In the current study, we conclude that gamma ray 
sterilization was the most effective nuclear technique for dealing with the threat posed by insects. The noticeable a product reaches the market once processing/or outer spoilage occurs. Although much research is done on the results of irradiation on food parts, studies on the results of irradiated manufacture on the overwhelming human population are nevertheless suitably reportable. Irradiation sterilization has remained the most effective and cost-effective method of managing dipterous insects over the years. An effective optimum dose of radiation is needed to provide potent males that are compatible with sexual union with wild females. The sterile males at these optimum doses were found to be potent and compatible in sexual union with wild females. The Sterile Insect is a type of insect that's also sterile. The technique where sterile insects are released competes with the mate method against fertile wild insects, resulting in a significant population reduction. Female mated with males irradiated at higher doses that were much more economical and resulted in higher egg/larvae abortion, fewer pupae, and a lower number of emerged adults from pupae than lower irradiation doses. The number of pupae created and adults emerged from pupae was lower in teams wherever sterile males were compared to fertile males and, also, the result was reciprocally proportional. On the other hand, teams containing a larger range of fertile male ratios than sterile males had a larger range.

\section{Declaration of Author}

This was my reviewed studied article without being conflict interest by the other. The contribution of the author was checked and announce for further nuclear researcher use of nuclear radiation in sterilization technique to control insect.

\section{Acknowledgements}

First of all, I would like thank physics department staffs to support critically and technically reviewed to success the present work.

\section{References}

[1] Munhenga et al. 2011. "Evaluating the potential of the sterile insecttechnique for malaria control: relative fitness andmating compatibility between laboratory colonizedand a wild population of Anopheles arabiensis fromthe Kruger National Park, South Africa." Parasites \& Vectors (South Africa) 4-208. $\mathrm{http}: / / \mathrm{www}$. parasitesandvectors.

[2] František Marec at al. (2019). Advances and Challenges of Using the Sterile InsectTechnique for the Management of Pest Lepidoptera. Insects, 10-371. doi: 10.3390/insects10110371

[3] Kiran Jonathan et al. 2020. "Egg Sterilisation of Irradiated Nezara viridula (Hemiptera: Pentatomidae)." insects (New Zealand) 11-564. doi: 10.3390/insects 11090564 .
[4] André Barretto et al. 2009. "Genetic enhancements to the sterile insect technique to control mosquito populations." AsPac J. Mol. Biol. Biotechnol. (São Paulo/SP, Brazil) 17 (3).

[5] Thiago Mastrangelo Et Al. 2009. "Assessment Of Differences Between X And $\Gamma$ Rays Inorder To Validate A New Generation Of Irradiators Forinsect Sterilization." International Nuclear Atlantic Conference - Piracicaba, Brazil.

[6] D. A. Kheirallah and L. M. El-Samada. 2020. "Midgut cells alteration in gamma-irradiated beetles (Blaps polycresta, Coleoptera: Tenebrionidae)." Braz. J. Biol, (Egypt) 80 (2): pp. 465-473. doi: doi.org/10.1590/1519-6984.217010.

[7] Ana Carolina Loro et al. 2018. "Quality parameters of tomatoes submitted to different doses of gamma radiation." (Brasil) 21.

[8] Aparecida, Kátia., 2012. "Sterilization by Gamma Irradiation." Sterilization by Gamma Irradiation, Brazil, doi: $10.5772 / 34901$.

[9] Jacob Urquidi et al. 2015. "The Development of New Radiation Protocols for Insect Sterilization Using Long Wavelength X- rays."RadiationPhysics (USA). doi: doi.org/10.1063/1.4927187.

[10] Demirbas-Uzel et al. 2018. "Combining paratransgenesis with SIT: impact of ionizing radiation on the DNAcopy number of Sodalis glossinidius intsetse flies." BMC Microbiology 2018, 18 (Suppl 1): 160 (Austria) 18 (1): 168. doi.org/10.1186/s12866-018-1283-8.

[11] Kavita Yadav et al. 2010. "Effect of Gamma Radiation on Survival and Fertility of Male Anopheles stephensi Liston, Irradiated as Pharate Adults." Journal of Ecobiotechnology 2/4: 06-10, 2010 (India) 2 (4): 6-10.

[12] Marc J. B. Vreysen et al. 2011. "Factory Tsetse Flies Must Behave Like Wild Flies: APrerequisite for the Sterile Insect Technique." Insect control (Montpellier, France) 5 (2).

[13] Thiago De A Et Al. 2007. "Use Of Gamma Radiation To Sterilize Ceratitis Capitata (Wied., 1824), Tsl Vienna 8 Strain, For Application Of Sterileinsect Technique In Brazil." International Nuclear Atlantic Conference (Brazil).

[14] Aydin S. T Et Al. 2012. "Effect Of Ionizing (Gamma) And Non-Ionizing (Uv) Radiation On The Development Of Trichogramma Euproctidis (Hymenoptera: Trichogrammatidae)." Arch. Biol. Sci., Belgrade (Turkey) 64 (1): 287-295. Doi: 10.2298/Abs1201287t.

[15] Waheed A. A. et al. 2019. "Biological Studies on the Effect of Gamma Radiation and Thermal Treatment on the Angoumois Grain Moth, Sitotroga cerealella (Olivier)." F. Toxicology \& Pest control (Egyptian) 11 (3): 29-36.

[16] Anil S Mohite et al. 2013. "Gamma Irradiation-Induced Parental and Inherited Sterility in Gram PodBorer, Helicoverpa armigera (Hubner) (Lepidoptera: Noctuidae)." Research Journal of Agricultural Sciences (India) 4 (1): 51-54. doi: 1068-12-3105-2012-11.

[17] Patama porn $\mathrm{K}$ et al. 2018. "Combined sterile insect technique and incompatible insect technique: sex separation and quality of sterile Aedes aegypti male mosquitoes released in a pilotpopulation suppression trial in Thailand." Parasites \& Vectors 11 (2). doi.org/10.1186/s13071-018-3214-9. 